\title{
DER SUEZ-KANAL UND DIE UNCTAD
}

Eine neue Welthandelskonferenz steht vor der Tür. Im Februar 1968 werden sich die Vertreter jener 120 Nationen und zwischenstaatlichen Organisationen, die bereits an der Konferenz für Handel und Entwicklung in Genf im Jahre 1964 teilnahmen, in Neu-Delhi versammeln. Delegierte neuer Staaten, die seit 1964 unabhängig geworden sind und Mitglieder der Vereinten Nationen wurden, werden das Aufgebot der Tagungsteilnehmer vergrößern. Eine umfangreiche Tagesordnung ist abzuhandeln; zahlreiche Punkte, bereits 1964 zur Diskussion gestellt und seitdem fortlaufend erörtert, sind immer noch aktuell. Auch dürfte die kommende Welthandelskonferenz manches von dem Charakter der voraufgegangenen Genfer Tagung übernehmen, nämlich einem „Konzil“ zu gleichen, auf dem Lehrmeinungen und Thesen verkündet werden, um daraus Forderungen herzuleiten und Verpflichtungen $z u$ begründen.

Der interessierte Beobachter des Zeitgeschehens ist allerdings überrascht, in wie geringem Maße die Tagesordnung für Neu-Delhi dem tatsächlichen Geschehen der allerjüngsten Vergangenheit, soweit davon der internationale $\mathrm{Handel}$ und die $\mathrm{Zu}$ sammenarbeit von Industriestaaten und Entwicklungsländern berührt wird, Rechnung trägt. Man sollte zum Beispiel meinen, daß die sich über Monate hinziehende Schließung des $\mathrm{S}$ u e $\mathrm{z}-\mathrm{K}$ a $\mathrm{n}$ a $\mathrm{l} \mathrm{s}$ das Thema Nr. 1 der Welthandelskonferenz zu sein hätte. Denn hier handelt es sich um ein Thema von eminent praktischer Bedeutung. Hier steht die fortdauernde Sperrung eines internationalen Verkehrsweges zur Debatte, welche nachweislich den von der Sperre betroffenen Ländern, insbesondere sogenannten Entwicklungsländern, einen Schaden verursacht, der in die Milliarden geht. Man hat errechnet, daß die Schließung des Suez-Kanals den Briten einen Jahresverlust von 600 Millionen Dollar in ihrer Devisen- und Zahlungsbilanz verursacht, errechnet nach den höheren Transportkosten für die eingeführten Güter, nach den gestiegenen Schiffahrtskosten und den höheren Preisen für Treibstoffe. Daß die durch „Suez“ bedingten Ausfälle sehr wesentlich zur Verschlechterung der Sterling-Position beigetragen haben, die Pfund-Abwertung also zu einem beachtlichen Teil auf das Konto "Suez" gebucht werden muß, sollte zu denken geben! Wenn die ostwärts von Suez gelegenen Staaten - von Sudan, Äthiopien, Somalia bis Ceylon, Indien, Indonesien - Bilanz ziehen und nachrechnen, wie ihre Devisenbilanz und ihre „earning power" durch die anhaltende Sperrung des Suez-Kanals betroffen wurden und weiterhin werden, so dürften auch sie zu erregenden Ziffern gelangen.

Man spricht oft von den „Terms of Trade“, die Länder ostwärts Suez" sollten in Neu-Dehli nicht in den allgemeinen Chor einstimmen, der das Lied von den bösen "Terms of Trade" vorträgt, sie sollten von ihren durch dieSperrung des Suez-Kanals willkürlich verschlechterten "Terms of Trade“ reden. Derartige Ausfälle, wie sie durch die Verlagerung der Transportwege verursacht wurden, lassen sich durch allgemeine Regelungen nicht ausgleichen. Hier sind Verluste entstanden und entstehen weiterhin, von denen unmittelbar diejenigen betroffen werden, die nicht mehr an dem großen internationalen Verkehrsweg liegen, sondern - man denke an Äthiopien oder Sudan - an die Peripherie gedrängt worden sind. In Genf brachte Dr. Paul Prebisch die höchst anfechtbare und leicht zu widerlegende Theorie der benachteiligten peripheren Staaten vor, in Neu-Delhi sollte man von der Wirklichkeit eine gewaltsam herbeigeführten Peripherie, einer veränderten Standortbedingung, sprechen. 
Man ist erstaunt, daß kein Entwicklungsland in den Vereinten Nationen die Suez-Frage unter diesem Aspekt zur Sprache gebracht hat. Unter Berufung auf den Artikel 1 der UN Charter ${ }^{1}$ wäre es ein leichtes gewesen, die Vereinten Nationen, unabhängig von der Frage des Israel-Ägypten-Komplexes, zu bewegen, der SuezKanal-Ơffnung für den neutralen internationalen Verkehr die gebührende Aufmerksamkeit zu widmen. In diesem Zusammenhang hätte man auf die 1964 von der Genfer Welthandelskonferenz einstimmig verabschiedete Empfehlung ${ }^{2}$ verweisen können:

Die Konferenz empfiehlt, daß zwischen den Regierungen geeignete Verfahren eingeführt werden, einschließlich der Bildung von Ausschüssen, die gegebenenfalls für erforderlich gehalten werden - entweder im Rahmen der Organisationen der Vereinten Nationen oder als Teil des Systems von Institutionen, die im Rahmen der Konferenz der Vereinten Nationen über Handel und Entwicklung geschaffen werden können - um das gegenseitige Verständnis und die Zusammenarbeit auf dem Gebiete der Schiffahrt zu fördern, und um wirtschaftliche Aspekte der Schiffahrt zu untersuchen, die ihnen möglicherweise zugewiesen werden ...

$\mathrm{Ob}$ die Frage der anhaltenden Sperrung des Suez-Kanals insofern ein heißes Eisen für dieKonferenz darstellt, als „Souveränitätsrechte“ berührt werden und die Genfer Konferenz 1964 das Prinzip der „Nichteinmischung in die internen Angelegenheiten anderer Länder" anerkannte, sollte nicht die von der Sperre Betroffenen davon abhalten, das Problem in Neu-Delhi mit Vordringlichkeit zur Diskussion zu bringen. Schließlich hat die Genfer Konferenz von 1964 einstimmig Grundsätze beschlossen, welche in bezug auf den Transit-Handel der sogenannten „land-locked countries" gerade im Hinblick auf eine bessere Entwicklung der Wirtschaft die Anerkennung von weitgehenden Rechten vorsehen. Die Konferenz der Vereinten Nationen über den Transithandel der Binnenstaaten von 1965, von der die VAR sich allerdings ferngehalten hat, hat diese Grundsätze dann in die Präambel der von allen beteiligten Staaten unterzeichneten Konvention über den Transithandel der Binnenstaaten aufgenommen. Einige Staaten, vor allem sogenannte Entwicklungsländer, verlangten sogar die Anerkennung der Grundsätze als geltendes Völkerrecht ${ }^{3}$.

Die Anerkennung des Rechts jedes von Landgebieten eingeschlossenen Staates auf freien Zugang zur See ist ein wesentlicher Grundsatz für die Ausweitung des internationalen Handels und der wirtschaftlichen Entwicklung4.

Um die Freiheit der Meere unter den gleichen Bedingungen wie Küstenstaaten genießen zu können, sollten küstenferne Staaten freien Zugang zur See haben. $\mathrm{Zu}$ diesem Zweck sollten Staaten, die zwischen der See und Staaten ohne Seeküste liegen, in gemeinsamer Úbereinkunft mit den letzteren und in Übereinstimmung mit bestehenden internationalen Vereinbarungen Schiffen des Staates ohne Seeküste gleiche Behandlung hinsichtlich des Zugangs und der Benützung von Seehäfen gewähren, wie ihren eigenen Schiffen oder den Schiffen irgendeines anderen Staates ${ }^{5}$.

\footnotetext{
1 Art. 1 (Ziele der VN) Die VN setzen sich folgende Ziele:

3. eine internationale Zusammenarbeit herbeizuführen, um internationale Probleme, wirtschaftlicher, sozialer, kultureller und humanitärer Art zu lösen ...

4. ein Mittelpunkt zu sein, in dem die Bemühungen der Nationen zur Verwirklichung dieser gemeinsamen Ziele aufeinander abgestimmt werden.

2 Zit. nach Bundesstelle für Außenhandelsinformation: Die Welthandelskonferenz in Genf, Köln 1964, S. 136.

- Vgl. Dieter Schröder, Der freie Zugang der Binnenstaaten zum Meer, Hamburg 1966, S. 18 ff.

- Grundsatz I., bei Schröder, a. a. O., S. 109.

- Grundsatz IIII., ebd.
} 
Es fragt sich, warum nicht die mit der "Ausweitung des internationalen Handels und der wirtschaftlichen Entwicklung " begründeten Forderungen für die Anerkennung von Rechten der „land-locked countries“ für die Durchfahrt durch den SuezKanal allgemein erhoben werden sollen.

Um die Offnung des Suez-Kanals und die Sicherheit der künftigen freien und ungehinderten Durchfahrt zu fördern, sollte in Neu-Delhi, besser schon vor der Konferenz, gesagt werden, wie sinnlos es ist, über Entwicklungs-Kooperation, über Grundsätze und Praktiken oder gar Leistungen zu sprechen, solange man nicht die Voraussetzungen für einen freien Handel und Verkehr durch Ausräumung von Hindernissen von der Art der Sperrung des Suez-Kanals schafft.

\section{DR. GÜNTHER JANTZEN}

\section{DIE LAGE HONGKONGS}

Die heutige britische Kronkolonie Hongkong besteht aus drei Teilen, die auch rechtlich getrennt zu betrachten sind:

1. Der Insel Hongkong ( $83 \mathrm{qkm}$ ), die im Jahre 1842 durch den Nankinger Friedensvertrag von China an Großbritannien „auf Ewigkeit“ abgetreten wurde';

2. der Halbinsel Kaulun (9 qkm), die im Jahre 1860 durch den Pekinger Friedensvertrag von China an Großbritannien „auf Ewigkeit“ abgetreten wurde ${ }^{2}$

3. und weiteren Festlandgebieten, den nördlich von Kaulun gelegenen sogenannten "New Territories“ (922 qkm), die durch einen Pachtvertrag Großbritannien auf 99 Jahre, d. h. bis zum. Jahre 1997 überlassen wurde 3 . In diesem Gebiet befinden sich heute nahezu die gesamte Industrie Hongkongs und die wichtigen öffentlichen Versorgungseinrichtungen der Kolonie, insbesondere die Wasserreservate.

Zusammen umfaßt das Gebiet, welches heute allgemein als Hongkong bezeichnet wird, eine Gesamtfläche von $1014 \mathrm{qkm}$.

Die genannten Verträge, die China unter Zwang mit anderen Mächten abgeschlossen hat, wurden von chinesischer Seite als ungleiche Verträge angesehen. Seit der Gründung der Republik China, bzw. des Sturzes der Ch'ing-Dynastie im Jahre 1911 hat sich China stets bemüht, diese Verträge zu beseitigen, um wieder seine eigene Souveränität zurückzugewinnen. Angesichts seiner damaligen schwachen militärischen und politischen Stellung war es China nicht gelungen, dieses Ziel aus eigener Kraft zu erreichen. Erst im Jahre 1941, als der Krieg zwischen Japan und den Alliierten ausgebrochen war, wurde China von Amerika und England als ein gleicher, ja sogar wichtiger Partner gegen Japan betrachtet, zumal China schon seit 1937 allein hart gegen Japan gekämpft hatte. Infolgedessen haben die amerikanische und die englische Regierung zu gleicher Zeit, nämlich am Vorabend des chinesischen Nationalfeiertages (10. Oktober) im Jahre 1942, ihre extraterritorialen Rechte in China für aufgehoben erklärt und den Abschluß neuer gleicher Verträge mit China angekündigt ${ }^{4}$.

\footnotetext{
1 Engl. Text des Vertrages: State Papers, Bd. 30, S. 389.

2 Engl. Text des Vertrages: State Papers, Bd. 50, S. 10.

3 Engl. Text des Vertrages: State Papers, Bd. 90, S. 17.

4 Text der britischen Erklärung: Keesing's Contemporary Archives 5396 B.
} 